\title{
ON UNIFORMLY APPROXIMABLE SIDON SETS
}

\author{
R. W. CHANEY
}

Let $G$ be a compact abelian group and let $\Gamma$ be the character group of $G$. Suppose $E$ is a subset of $\Gamma$. A trigonometric polynomial $f$ on $G$ is said to be an $E$-polynomial if its Fourier transform $\hat{f}$ vanishes off $E$. The set $E$ is said to be a Sidon set if there is a positive number $B$ such that $\sum_{\lambda \in E}|\hat{f}(\lambda)| \leqq B\|f\|_{u}$ for all $E$-polynomials $f$; here, $\|f\|_{u}$ $=\sup \{|f(x)|: x \in G\}$. In this note we shall discuss a certain class of Sidon sets-the class of all "uniformly approximable" Sidon sets.

A Sidon set $E$ is said to be uniformly approximable if the characteristic function of $E$ is in the uniform closure of the algebra of FourierStieltjes transforms of Radon measures on $G$. The principal result in this note is a theorem which gives a list of characterizations of uniformly approximable Sidon sets. These are somewhat akin to characterizations for Sidon sets given by Hewitt and Zuckerman [2, 2.1 and 8.5$]$ and by Rudin ([7] and [6, 5.7]). Also, we shall propose several alternative characterizations and show (except in one case) that these would-be characterizations are false.

Stečkin [8, p. 394] established a sufficient condition for Sidon sets, which has been generalized successively by Hewitt and Zuckerman [2], Rudin [7], and Rider [5]. Evidently all known Sidon sets are finite unions of Sidon sets which satisfy Rider's condition and it will be shown that all of these finite unions are uniformly approximable Sidon sets.

Ramirez [3] has studied subsets of $\Gamma$ whose characteristic functions are in the uniform closure of the algebra of Fourier-Stieltjes transforms. Among the results presented in [3] are several theorems about uniformly approximable Sidon sets.

We must establish certain conventions about notation. Throughout the paper $G$ denotes an arbitrary compact abelian group and $\Gamma$ denotes the character group of $G$. We denote the measure algebras of $G$ and $\Gamma$ by $M(G)$ and $M(\Gamma)$ respectively and the group algebra of Haar-integrable functions on $G$ by $L_{1}(G)$. We denote by $C(G)$ the algebra of all complex-valued continuous functions on $G$. If $A \subset \Gamma$, then $A^{\prime}$ is the complement of $A, \xi_{A}$ is the characteristic function of $A$ in $\Gamma$, and $C_{0}(A)$ denotes the set of all complex-valued functions on $A$ which vanish at infinity. For $A \subset D \subset \Gamma$, we let $C(D, A)$ be the set of

Presented to the Society, June 15, 1968; received by the editors January 5, 1968. 
all bounded, complex-valued functions on $D$ which vanish on $D \cap A^{\prime}$; if $A=D$ we put $C(D, A)=C(D)$. Finally, $(x, \gamma)$ is the value which the character $\gamma$ in $\Gamma$ assumes at the point $x$ in $G$.

Definition. Suppose $E \subset \Gamma$. Then $E$ is said to be a uniformly approximable Sidon set if it is a Sidon set and if for every $\epsilon>0$ there exists a measure $\mu$ in $M(G)$ such that $\left\|\hat{\mu}-\xi_{E}\right\|_{u}<\epsilon$.

Theorem. Suppose $E \subset \Gamma$. These statements about $E$ are equivalent:

(1) $E$ is a uniformly approximable Sidon set.

(2) For every $\epsilon>0$ and for every $f$ in $C(\Gamma, E)$ there is a measure $\mu$ in $M(G)$ such that $\|\hat{\mu}-f\|_{u}<\epsilon$.

(3) For every $\epsilon>0$ and for every $f$ in $C(E)$ there is a measure $\mu$ in $M(G)$ such that $\hat{\mu}=f$ on $E$ and $|\hat{\mu}|<\epsilon$ on $E^{\prime}$.

(4) For every $\epsilon>0$ there exists $B>0$ such that if $f$ is in $C(E)$ then there exists $\mu$ in $M(G)$ such that $\hat{\mu}=f$ on $E,|\hat{\mu}| \leqq \epsilon\|f\|_{u}$ on $E^{\prime}$ and $\|\mu\| \leqq B\|f\|_{u}$.

(5) For every $\epsilon>0$ there exists $B>0$ such that if $\phi$ is in $C_{0}(E)$ there exists $f$ in $L_{1}(G)$ such that $\hat{f}=\phi$ on $E,|\hat{f}| \leqq \epsilon\|\phi\|_{u}$ on $E^{\prime}$, and $\|f\|_{1}$ $\leqq B\|\phi\|_{u}$.

(6) For every $\epsilon>0$ there exists $B>0$ such that if $g$ is a trigonometric polynomial in $C(G)$ then

$$
\sum_{\gamma \in E}|\hat{g}(\gamma)| \leqq B \sup \left\{\left|\sum_{\gamma \in E} \hat{g}(\gamma)(x, \gamma)\right|: x \in G\right\}
$$

and there is also a trigonometric polynomial $h$ in $C(G)$ such that $\hat{h}=\hat{g}$ on $E,|\hat{h}| \leqq \epsilon|\hat{g}|$ on $E^{\prime}$, and $\|h\|_{1} \leqq B\|g\|_{1}$.

(7) If $f$ is in $C(\Gamma, E)$ and if $\left\{\lambda_{n}\right\}$ is any sequence of measures in $M(\Gamma)$ such that $\left\|\lambda_{n}\right\| \leqq 1$ for each $n$ and $\lim _{n} \hat{\lambda}_{n}(x)=0$ for every $x$ in $G$ then $\lim _{n} \int_{\Gamma} f d \lambda_{n}=0$.

Proof. $(1) \Rightarrow(2)$. Suppose that $E$ is a uniformly approximable Sidon set. Let $\epsilon>0$ be given and suppose $f$ is in $C(\Gamma, E)$. There exist $\nu$ and $\sigma$ in $M(G)$ such that $\hat{\nu}=f$ on $E$ and $\left\|\hat{\sigma}-\xi_{E}\right\|_{u}<\epsilon(\|\nu\|+1)^{-1}$. If $\mu=\nu * \sigma$ then clearly $\|\hat{\mu}-f\|_{u}<\epsilon$.

$(2) \Rightarrow(3)$. Suppose (2) holds. According to $[6,5.7 .4], E$ is a Sidon set. Hence there is a number $B>0$ such that if $g$ is any function in $C(E)$ then there is a measure $\mu$ in $M(G)$ so that $\hat{\mu}=g$ on $E$ and $\|\mu\| \leqq B\|g\|_{u}$ (see the proof of $[6,5.7 .3]$ ).

Now let $\epsilon>0$ and $f$ in $C(\Gamma, E)$ be given. By (2) there exists $\sigma$ in $M(G)$ such that $\|\hat{\sigma}-f\|_{u}<\min \left\{1, \epsilon B^{-1}\right\}$. Put $A=\{\gamma \in E:|f(\gamma)| \geqq 2\}$ and $D=E \cap A^{\prime}$. Define $h$ on $E$ by setting $h(\gamma)=f(\gamma) / \hat{\sigma}(\gamma)$ if $\gamma$ is in $A$ and $h(\gamma)=1$ if $\gamma$ is in $D$. It is easy to check that $h$ is in $C(E)$ and $\|h\|_{u} \leqq 2$. Consequently there exists $\nu$ in $M(G)$ such that $\hat{\nu}=h$ on $E$ and $\|\nu\| \leqq 2 B$. Define $g$ on $E$ by putting $g(\gamma)=0$ if $\gamma$ is in $A$ and 
$g(\gamma)=f(\gamma)-\hat{\sigma}(\gamma)$ if $\gamma$ is in $D$. Then $\|g\|_{u} \leqq\|f-\hat{\sigma}\|_{u}$ and so there exists $\tau$ in $M(G)$ such that $\hat{\tau}=g$ on $E$ and $\|\tau\|<\epsilon$. If $\mu=\sigma * \nu+\tau$ then it is easy to verify that $\hat{\mu}=f$ on $E$ and $|\hat{\mu}|<3 \epsilon$ on $E^{\prime}$.

$(3) \Rightarrow(4)$. Suppose (3) holds. Since $E$ is a Sidon set $[6,5.7 .3]$ there is a number $B_{1}>0$ such that to each $f$ in $C(E)$ there corresponds $\nu$ in $M(G)$ such that

$$
\hat{\nu}=f \quad \text { on } \quad E, \quad\|\nu\| \leqq B_{1}\|f\|_{u} .
$$

Let $\epsilon>0$ be given. By (3) there exists $\sigma$ in $M(G)$ such that $\hat{\sigma}=1$ on $E$ and $|\hat{\sigma}|<\epsilon B_{1}^{-1}$ on $E^{\prime}$. Put $B=B_{1}\|\sigma\|$. If $f$ is in $C(E)$ then there exists $\nu$ in $M(G)$ such that (8) holds. The measure $\mu=\nu * \sigma$ has the desired properties.

$(4) \Rightarrow(5)$. Suppose (4) holds. Since $E$ is a Sidon set there is a number $B_{1}>0$ such that to each $\phi$ in $C_{0}(E)$ there corresponds $g$ in $L_{1}(G)$ such that $g=\phi$ on $E$ and $\|g\|_{1} \leqq B_{1}\|\phi\|_{u}$. The rest of the proof resembles closely the proof that (3) implies (4); we omit further details.

$(5) \Rightarrow(6)$. Suppose (5) holds. Let $\epsilon>0$ be given; there is a number $B>0$ with the properties listed in (5). Let $g$ be a trigonometric polynomial in $C(G)$. We can proceed as in the proof of $[6,5.7 .3]$ to show that (A) must hold. Also, there exists $f$ in $L_{1}(G)$ such that $\hat{f}(\gamma)=1$ if $\boldsymbol{\gamma}$ is in $E$ and $\hat{g}(\gamma) \neq 0, \hat{f}(\gamma)=0$ if $\gamma$ is in $E$ and $\hat{g}(\gamma)=0,|\hat{f}| \leqq \epsilon$ on $E^{\prime}$, and $\|f\|_{1} \leqq B$. Put $h=g * f$. Then $g$ is a trigonometric polynomial; moreover, $\hat{h}=\hat{g}$ on $E,|\hat{h}| \leqq \epsilon|\hat{g}|$ on $E^{\prime}$, and $\|h\|_{1} \leqq B\|g\|_{1}$.

$(6) \Rightarrow(1)$. Suppose (6) holds. Condition (6-A) shows at once that $E$ must be a Sidon set. It remains to show that the Sidon set $E$ is uniformly approximable. Let $\varepsilon$ be the collection of all finite subsets of $E$; we direct $\varepsilon$ by inclusion. For each $F$ in $\varepsilon$ there is, by $[6,2.6 .8]$, a trigonometric polynomial $g_{F}$ such that $\hat{g}_{F}=1$ on $F$ and $\left\|g_{F}\right\|_{1} \leqq 2$.

Now let $\epsilon>0$ be given. There is a $B>0$ which has the properties listed in (6). Hence it is true that to each $F$ in $\varepsilon$ there corresponds a trigonometric polynomial $h_{F}$ such that $\hat{h}_{F}=\hat{g}_{F}$ on $E,\left|\hat{h}_{F}\right| \leqq \epsilon\left|\hat{g}_{F}\right|$ on $E^{\prime}$, and $\left\|h_{F}\right\|_{1} \leqq 2 B$. We let $\lambda$ denote normalized Haar measure on $G$ and consider the net $\left\{h_{F}(x) d \lambda(x): F \in \varepsilon\right\}$ of measures in $M(G)$. We may assume that this net actually converges to some $\mu$ in $M(G)$ in the weak-* topology. It is easy to see that $\hat{\mu}=1$ on $E$. If $\gamma$ is in $E^{\prime}$ then $|\hat{\mu}(\gamma)| \leqq \sup \left\{\left|\hat{h}_{F}(\gamma)\right|: F \in \mathcal{E}\right\} \leqq \epsilon \sup \left\{\left|\hat{g}_{F}(\gamma)\right|: F \in \mathcal{E}\right\} \leqq 2 \epsilon$. Hence $\left\|\hat{\mu}-\xi_{E}\right\|_{u} \leqq 2 \epsilon$.

We have proved that (1)-(6) are all equivalent.

$(2) \Leftrightarrow(7)$. Ramirez has proved $[4,1.9]$ that a function $f$ in $C(\Gamma)$ is in the uniform closure of $M(G)^{\wedge}=\{\hat{\mu}: \mu \in M(G)\}$ if and only if it is true that whenever $\left\{\lambda_{n}\right\}$ is any sequence of measures in $M(\Gamma)$ such that $\left\|\lambda_{n}\right\| \leqq 1$ for each $n$ and such that $\lim _{n} \lambda_{n}(x)=0, x \in G$, then 
$\lim _{n} \int_{\mathrm{r}} f d \lambda_{n}=0$; this result is a generalization of a result due to $\mathrm{A}$. Beurling and E. Hewitt (unpublished). It follows at once from this characterization that (2) and (7) are equivalent.

Note. Perhaps the most interesting part of the theorem is the assertion that (7) implies each of the other conditions.

REMARK 1. If the subset $E$ of $\Gamma$ is a uniformly approximable Sidon set then so is any subset of $E$ (and, so is $-E$ ). It follows (from (2), for example) that the union of any two uniformly approximable Sidon sets is again a uniformly approximable Sidon set.

REMARK 2. Let $E$ be a countable subset of $\Gamma$. Enumerate the elements of $E$ as $\gamma_{1}, \gamma_{2}, \gamma_{3}, \cdots$. For each positive integer $s$ and each character $\gamma$ in $\Gamma$ let $R_{s}(E, \gamma)$ denote the number of representations of $\gamma$ in the form

$$
\gamma= \pm \gamma_{n_{1}} \pm \gamma_{n_{2}} \pm \cdots \pm \gamma_{n_{s}}, \quad n_{1}<n_{2}<\cdots<n_{s}
$$

The identity element of $\Gamma$ is denoted by 0 .

Rider has shown [5, Theorem 1.5] that if there is a number $B>0$ such that $R_{s}(E, 0) \leqq B^{s}$ for $s=1,2, \cdots$, then $E \cup-E$ is a uniformly approximable Sidon set; it follows that $E$ must itself be a uniformly approximable Sidon set.

It seems to be true that all known Sidon sets are finite unions of Sidon sets which satisfy this sufficient condition given by Rider. Seemingly then, all known Sidon sets are uniformly approximable.

Remark 3. Suppose $E \subset \Gamma$. Then $E$ is a Sidon set if and only if for every function $g$ in $C(E)$ there is a measure $\mu$ in $M(G)$ such that $\hat{\mu}=g$ on $E$, or, if and only if for every $\phi$ in $C_{0}(E)$ there exists $f$ in $L_{1}(G)$ such that $\hat{f}=\phi$ on $E$. By analogy with (3) it might be conjectured that $E$ is a uniformly approximable Sidon set if and only if

$\left(5^{\prime}\right)$ for every $\epsilon>0$ and every $\phi$ in $C_{0}(E)$ there exists $f$ in $L_{1}(G)$ such that $\hat{f}=\phi$ on $E$ and $|\hat{f}|<\epsilon$ on $E^{\prime}$.

But $\left(5^{\prime}\right)$ actually holds for every Sidon set $E$. Indeed if $\phi$ is in $C_{0}(E)$, $g$ is in $L_{1}(G)$, and $\hat{g}=\phi$ on $E$ then we can obtain $f$ in $L_{1}(G)$ so that $\hat{f}=\phi$ on $E$ and $|\hat{f}|<\epsilon$ on $E^{\prime}$ by subtracting a suitably chosen trigonometric polynomial from $g$.

REMARK 4. We shall discuss certain conditions (listed below as (i)-(iv)) which appear to be more stringent than properties (3)-(6) and we shall show that these conditions are in fact too stringent to be satisfied by all uniformly approximable Sidon sets.

First, we must introduce more notation. Suppose $A \subset \Gamma$. We denote by $C_{A}(G)$ the set of all functions $f$ in $C(G)$ for which $\hat{f}(\gamma)=0$ for all $\gamma$ in $A^{\prime}$. Let $M[A]$ (or, $L_{1}[A]$ ) be the set of all $s$ in $M(G)$ (or, $L_{1}(G)$ ) such that $\hat{s}(\gamma)=0$ for all $\gamma$ in $A^{\prime}$. 
Given $E \subset \Gamma$, the following four statements about $E$ are equivalent:

(i) There is a number $B>0$ such that $\sum_{\gamma \in E}|\hat{g}(\gamma)| \leqq B\|g\|_{u}$ for every trigonometric polynomial $g$ in $C(G)$.

(ii) There is a number $B>0$ such that $\sum_{\gamma \in E}|\hat{g}(\gamma)| \leqq B\|g\|_{u}$ for every $g$ in $C(G)$.

(iii) To each $\phi$ in $C(E)$ there corresponds $\mu \in M[E]$ such that $\hat{\mu}=\phi$ on $E$.

(iv) To each $\phi$ in $C_{0}(E)$ there corresponds $f \in L_{1}[E]$ such that $\hat{f}=\phi$ on $E$.

The proof of the assertion that (i)-(iv) are equivalent is very similar to the proof in $[6,5.7 .3]$; a proof may be based upon the fact that $M[E]$ is the adjoint of the quotient space $C(G) / C_{E^{\prime}}(G)$ (see $[1,1.4])$. Statements (i)-(iv) are clearly equivalent to

(v) $E$ is a Sidon set and $\xi_{E}$ is in $M(G)^{\wedge}$.

Now suppose $E$ satisfies (v). There exists $\mu$ in $M(G)$ such that $\hat{\mu}=\xi_{E}$. But, since $E$ is a Sidon set and $\mu$ is in $M[E], \mu$ must be absolutely continuous; indeed, it can be shown that $\hat{\mu}$ must be the Fourier transform of a function in $\bigcap_{1 \leqq p<\infty} L_{p}(G)[6,5.7 .7$ and 2.6.8]. Therefore $\xi_{E}=\hat{\mu}$ is in $C_{0}(\Gamma)$ and so $E$ is finite.

Hence only finite sets $E$ satisfy (i)-(v) and so conditions (i) $-(\mathrm{v})$ do not hold for all uniformly approximable Sidon sets.

I wish to thank the referee for suggesting many improvements in the paper.

\section{REFERENCES}

1. R. W. Chaney, Sets in discrete abelian groups which support Fourier-Stieltjes transforms only of absolutely continuous measures, unpublished.

2. E. Hewitt and H. S. Zuckerman, Some theorems on lacunary Fourier series, with extensions to compact groups, Trans. Amer. Math. Soc. 93 (1959), 1-19.

3. D. E. Ramirez, Uniform approximation by Fourier-Stieltjes coefficients, Proc. Cambridge Philos. Soc. 64 (1968), 615-623.

4. - Uniform approximation by Fourier-Stieltjes transforms, Proc. Cambridge Philos. Soc. 64 (1968), 323-333.

5. D. Rider, Gap series on groups and spheres, Canad. J. Math. 18 (1966), 389-398.

6. W. Rudin, Fourier analysis on groups, Wiley, New York, 1962.

7. —_, Trigonometric series with gaps, J. Math. Mech. 9 (1960), 203-228.

8. S. B. Stečkin, On absolute convergence of Fourier series, Izv. Akad. Nauk SSSR Ser. Mat. 20 (1956), 385-412.

University of California, Santa Barbara 